\title{
El sesgo economicista o social como evidencia del Curriculum Oculto en los grados de Finanzas y Contabilidad y Relaciones laborales y Recursos Humanos de la Universidad de Sevilla
}

\author{
The economicist or social bias as evidence of the Hidden \\ Curriculum in the degrees of Finance and Accounting and \\ Labor Relations of the University of Seville.
}

Francisco Serrano Domínguez (fserrano@us.es)

Departamento de Contabilidad y Economía Financiera. Universidad de Sevilla, (Sevilla)

ORCID: 0000-0003-2455-1520

\section{$M^{a}$ Carmen Aguilar del Castillo (carmenag@us.es)}

Departamento de Derecho del Trabajo y de la Seguridad Social. Universidad de Sevilla, (Sevilla)

ORCID: 0000-0002-5994-2655

https://dx.doi.org/10.12795/EDUCADE.2021.i12.04

\begin{abstract}
RESUMEN: Generalmente, el curriculum oculto pretende representar un trasfondo de normas, valores y reglas latentes en el proceso formativo, que los estudiantes han de asumir y adoptar para desempeñar convincentemente un papel social. Durante la etapa de formación primaria y secundaria, el curriculum oculto se centra, principalmente, en iniciar a los estudiantes en el desarrollo de determinadas competencias transversales Muchas de ellas se continúan potenciando en los estudios superiores. El trabajo que presentamos surge como consecuencia de la aparición en el grado de Finanzas y Contabilidad y del grado de Relaciones Laborales y Recursos Humanos de sendas asignaturas optativas especialmente singulares pues son impartidas por distintas áreas de conocimiento (Contabilidad y Derecho del Trabajo) en las que, desde sus inicios se ha observado la influencia del curriculum oculto, transmitido a los estudiantes durante su paso por la titulación, lo que se demuestra en la diferente respuesta que dan los alumnos a la resolución de problemas idénticos cuando disponen, aunque con distintos niveles de intensidad, de la misma información y formación. Se observa un claro corte economicista para los estudiantes de Finanzas y Contabilidad, en el que prima la eficacia, la eficiencia y la maximización del beneficio mientras que los estudiantes del Grado de Relaciones Laborales muestran una visión más social donde las relaciones laborales y su defensa cobran un especial protagonismo.
\end{abstract}

PALABRAS CLAVE: Curriculum Formal; Curriculum Real; Curriculum Oculto; Desarrollo Curricular; Estudios de Grado; Educación Superior; Asignaturas Optativas; Estudio de Caso.

Artículo de investigación. Recibido: 09-02-21 - Revisiones 07-09-21; 19-11-21 - Aceptado: 02-12-21

Licencia Creative Commons BY NC ND · $2021 \cdot$ Universidad de Sevilla - AECA

ABSTRACT: Generally, the hidden curriculum pretends to represent a background of latent norms, values and rules in the formative process, which students have to assume and adopt in order to convincingly play a social role. During the primary and secondary education stage, the hidden curriculum focuses mainly on initiating students in the development of certain transversal competences. Many of them continue to be promoted in higher studies. The work that we present arises as a consequence of the appearance in Finance and Accounting degree and the Labor Relations and Human Resources degree of two especially unique elective subjects since they are 
taught by different areas of knowledge (Accounting and Labor Law) in which, Since its inception, the influence of the hidden curriculum has been observed, transmitted to students during their passage through the degree, which is demonstrated in the different response that students give to solving identical problems when available, although with different levels of intensity, of the same information and training. A clear economic cut is observed for Finance and Accounting students, in which effectiveness, efficiency and profit maximization prevail while the students of the Degree in Labor Relations show a more social vision where labor relations and its defense take on a special role.

KEYWORDS: Curriculum, Real Curriculum; Hidden Curriculum; Curricular development; Graduate Studies; Higher Education; Elective Subjects; Case Study.

\section{INTRODUCCIÓN}

España, en su proceso de integración en materia educativa con el resto de la Unión Europea, se adhirió al Espacio Europeo de Educación Superior (EEES) emprendiendo un camino que se concreta en la creación de las titulaciones de grado.

Siguiendo el espíritu del EEES el alumno es el centro sobre el que pivota toda la educación, de modo que el profesor adquiere un rol en el que debe predominar la orientación del estudiante, así como ser el inculcador de las habilidades, capacidades y competencias propias de los profesionales que ejercen la profesión correspondiente a su titulación. Este concepto supera al tradicional en el que la educación se entendía como un proceso instructivo en el que los alumnos eran receptores de conocimientos que vertían sobre ellos profesores expertos en una materia determinada (Carrillo, 2009).

La integración de las titulaciones al EEES se materializa a través de los planes de estudios descritos de manera documental (con independencia del soporte utilizado), a través de descriptores, planes y programas necesarios para alcanzar el objetivo de obtener una determinada titulación. Es lo que se denomina Curriculum Oficial o Formal (Posner, 2005). La legitimidad del Curriculum Formal se basa en la racionalidad y coherencia de su planteamiento que va desde su fundamentación hasta las operaciones que deben ponerse en práctica y que lógicamente se sostienen por una estructura académica, administrativa, legal y económica (M. Casarini, 1999).

En teoría, cualquier estudiante, con independencia del centro en el que curse sus estudios, debería recibir la misma formación ya que todos los centros deberían impartir el mismo Curriculum Formal.

Cuando tratamos de trasladar el Curriculum Formal a los proyectos docentes de cada asignatura, comienzan a manifestarse diferencias en el modo de abordarlo lo que distingue, de manera explícita, los contenidos y competencias (genéricas, transversales y específicas) que el alumno debería haber adquirido al superar la asignatura. Por tanto, existe una diferencia entre el Curriculum Formal y lo que realmente el alumno va a recibir - Curriculum Real (también llamado Curriculum vivido) (Martha Casarini, 2004) puesto que el proyecto docente expresa de forma concreta el nivel de profundidad de los contenidos fijados en el Curriculum Formal, adaptándolos tanto a las circunstancias específicas en la que se va a desarrollar la docencia (nivel de los estudiantes, posibilidad de uso de las TIC, infraestructura de las aulas, etc.) como a las circunstancias ambientales (nivel socioeconómico, cultural, ideológico, etc.) en el que se va a desarrollar el proceso de Enseñanza-Aprendizaje.

En contraposición al concepto de Curriculum Formal, durante la aplicación del Curriculum Real, forma parte del proceso de Enseñanza-Aprendizaje la asunción de ciertas prácticas institucionales que derivan de enseñanzas encubiertas, latentes y que no están contempladas ni en los planes de estudio ni en los proyectos docentes y que, sin embargo, puede ocasionar cambios en la conducta de los estudiantes o en la forma 
de razonar y afrontar la solución de problemas. Estaríamos hablando de un tercer pilar sobre el que se basa la formación del estudiante: el Curriculum Oculto.

En el presente trabajo queremos demostrar la existencia de un Curriculum Oculto en los estudiantes que cursan el grado de Finanzas y Contabilidad y el Grado de Relaciones Laborales y Recursos Humanos de la Universidad de Sevilla por comparación de los resultados de experiencias similares. Para ello, nos basaremos en la experiencia realizada en las asignaturas optativas "Gestión de Costes Laborales", impartida en el Grado de Finanzas y Contabilidad (GCL-FICO) y en la asignatura "Contenidos Económicos para la Negociación Colectiva" (CENC-RRLL) impartida en el Grado de Relaciones Laborales y Recursos Humanos.

El trabajo que se presenta tiene la siguiente estructura: tras la presente introducción, procederemos a profundizar sobre el concepto de Curriculum Oculto, sus diferentes acepciones y características, centrándonos fundamentalmente en los estudios científicos realizados relacionados con el ámbito universitario. Con posterioridad se describe el caso objeto de estudio para, finalmente, extraer una serie de conclusiones.

\section{EL CURRICULUM OCULTO}

Dentro de este apartado vamos a adentrarnos en el concepto de Curriculum Oculto, sus diferentes acepciones y características para, finalmente, detenernos en los estudios que se han realizado relacionados con el ámbito universitario.

\subsection{Evolución del concepto de Curriculum Oculto. Características.}

El Curriculum Oculto no es un concepto reciente, ya que Durkheim (1961) hablaba del Curriculum Oculto, aunque sin acuñar directamente ese término, al observar que los estudiantes aprendían más de lo que aparecía especificado en los planes de estudio y en los manuales de los profesores (Durkheim, 2002).

Quizás sea Philip Jackson (1968), uno de los primeros en utilizar la expresión Curriculum Oculto, definiéndolo como "el conjunto de normas, costumbres, creencias, lenguajes y símbolos que se manifiestan en la estructura y el funcionamiento de una institución. Sin pretenderlo de manera reconocida, el currículum oculto constituye una fuente de aprendizajes para todas las personas que integran la organización. Los aprendizajes que se derivan del currículum oculto se realizan de manera osmótica, sin que se expliciten formalmente ni la intención ni el mecanismo o procedimiento cognitivo de apropiación de significados" (Jackson, 1968, pág 25).

El Curriculum oculto envuelve todo lo que ocurre en el centro de enseñanza. A través de las observaciones en las aulas de la escuela primaria pública, Jackson identificó rasgos de la vida del aula que eran inherentes a las relaciones sociales de la escolarización. Observó cómo había valores, disposiciones y expectativas sociales y de comportamiento que conllevaban premios para los estudiantes que las observaban siendo, por tanto, el aprendizaje de "lo que se esperaba" una de las características del currículo oculto.

Jackson (1968) sostuvo que el currículum oculto enfatizaba habilidades específicas tales como:

Aprender a esperar en silencio.

- El ejercicio de la moderación.

- Intentar mantenerse ocupado al finalizar un trabajo.

- Cooperar, mostrando lealtad a los profesores y compañeros. 
- Ser ordenado y puntual.

Conducirse durante la permanencia en el centro con cortesía.

Estas características de la vida escolar, y requisitos de conformidad a las expectativas institucionales, tenían poco que ver con las metas educativas, pero resultaban esenciales para la progresión satisfactoria a través de la escuela.

Casi al mismo tiempo, Dreeben (1968) argumentaba que la estructura de la vida familiar por sí sola no podría preparar adecuadamente a los niños para el mundo de los adultos, sosteniendo que la experiencia de la educación formal no sólo se enseña a través del currículo explícito, sino que indirectamente transmitía valores a los estudiantes como la independencia y la lucha por alcanzar objetivos y que éstos resultaban útiles para su posterior incorporación en la sociedad adulta.

Estas obras fundamentales de Durkheim, Parsons, Jackson y Dreeben se recogen dentro de lo que se conoce en el campo de la sociología como la teoría del consenso (Margolis, 2002). Esta teoría establece las bases para la definición general del currículo oculto como los elementos de socialización que tienen lugar en la escuela, pero que no forman parte del contenido curricular formal, como por ejemplo ganar tiempo haciendo actividades mientras se espera al profesor. Estos elementos de socialización incluyen normas, valores y sistemas de creencias incorporadas en el plan de estudios, la escuela y la vida del aula, impartidas a los estudiantes a través de las rutinas diarias, los contenidos curriculares y las relaciones sociales.

Influenciado por el marxismo, algunas ramas de la teoría educativa posteriores se hicieron más críticas sobre la forma en que las escuelas sirven al capitalismo y al estado y la reproducción de la desigualdad, incluyendo la de clase social (Apple, 1991; Riseborough, 1998, 2006; Torres, 1998, Celis y Moreno, (2017), la de raza (Apple y King, 2014; Flecha, 1999; Torres, 1998) y la de género (Bonal, 2005; Giroux, 1992; E. Moreno, 2000; Santos Guerra, 1996; Torres, 1998).

El examen más influyente del proceso por el cual las escuelas reproducen estos intereses dominantes fue la escolarización en la América capitalista por Samuel Bowles y Herbert Gintis (1976). En lo que llamaron la "tesis de la correspondencia", estos economistas demostraron la relación entre las normas de comportamiento de la escuela y el mantenimiento del sistema capitalista. Estos demostraron que, a través de las escuelas formales y ocultos bajo los planes de estudio, se reproducen las relaciones sociales necesarias para mantener el capitalismo: la competencia y la evaluación, las divisiones jerárquicas de la mano de obra, la autoridad burocrática, el cumplimiento y la naturaleza fragmentada y alienada del trabajo. Estos autores señalaron que la reproducción de estas habilidades y actitudes a través del sistema educativo prepara a los estudiantes para futuros roles de trabajo estratificados. Bajo la forma de organización del aula y la evaluación de los estudiantes se transmite un sistema de mensajes que los empuja a adoptar rasgos como los de la puntualidad, la docilidad, la limpieza y la conformidad.

El mensaje exacto varía en función de la clase social de la comunidad que se encuentra alrededor de la escuela. Los estudiantes en las escuelas de clase media alta llegaron algunos mensajes sobre la internalización de los objetivos a lograr, mientras que los de las escuelas de la clase trabajadora ensayado las conductas apropiadas para la baja cualificación, trabajo de baja autonomía.

Para estos autores, que ratificaron su estudio posteriormente (Bowles y Gintis, 2002), el currículo oculto es el proceso de inculcar estos comportamientos a través de las características naturales y cotidianas de la vida escolar atendiendo las demandas de las instituciones más potentes y los grupos sociales dominantes. 


\subsection{Características del Curriculum Oculto}

A efectos de comprender si estamos en presencia en un aprendizaje basado en el Curriculum Oculto, éste debe reunir una serie de rasgos característicos que lo definan. En la literatura revisada, la mayor parte de autores coincide en esas características definitorias. Siguiendo a Etkin (1993) estas características son:

a) Es subrepticio, es decir, influye de manera no manifiesta, aunque no por eso es menos efectivo. A través de la observación, de la repetición automática de comportamientos, del cumplimiento de las normas, de la utilización de los lenguajes, de la asunción de las costumbres, acabamos asimilando una forma de ser y de estar en la cultura generada por la institución.

b) Es omnipresente porque actúa en todos los momentos y en todos los lugares. De ahí su importancia y su intensidad. La forma de organizar el espacio y de distribuir el tiempo está cargada de significados, la naturaleza de las relaciones está marcada por los papeles que se desempeñan, las normas están siempre vinculadas a una concepción determinada de poder... Cuando estamos en una organización permanecemos inmersos en su clima.

c) Es omnímodo, porque reviste múltiples formas de influencia. Se asimilan significados a través de las prácticas que se realizan, de los comportamientos que se observan, de las normas que se cumplen, de los discursos que se utilizan, de las contradicciones que se viven, de los textos que se leen, de las creencias que se asumen.

d) Es reiterativo, como lo son las actividades que se repiten de manera casi mecánica en una práctica institucional que tiene carácter rutinario. Se entra a la misma hora, se hacen las mismas cosas, se mantienen las mismas reglas, se perpetúan los mismos papeles.

e) Es invaluable, es decir, que no se repara en los efectos que produce, no se evalúa el aprendizaje que provoca, no se valora las repercusiones que tiene. Se evalúa el currículum explícito, tanto en los aprendizajes que ha provocado en los alumnos/as como de manera global a través de las evaluaciones de Centros. Pero no se tiene en cuenta todo lo que conlleva la forma de estructuración, funcionamiento y relación que constituye la cultura de la institución.

Estas características brindan al Curriculum Oculto una eficacia poderosa ya que el individuo no es consciente de su influencia, no somete a revisión crítica ese influjo y hace que no se pueda defender fácilmente de sus perversiones. Esto no quiere decir que todos los componentes del aprendizaje, que se derivan del Curriculum Oculto, sean negativos también se encuentran dentro de él algunas prácticas institucionales que se asientan sobre la racionalidad y la ética (Etkin, 1993; Silverwood et al., 2017).

\subsection{El Curriculum Oculto en la Universidad}

El concepto de currículo oculto se asocia generalmente con la educación primaria. Este supuesto es fácilmente explicable puesto la mayor parte de la investigación desarrollada en torno a este concepto se ha enfocado en este nivel de enseñanza, probablemente porque en ese plano el impacto en forma de alta socialización que pueda generar en los estudiantes sea mayor.

Sin embargo, el Currículum oculto se da en todas las formas de la educación institucionalizada (Illich, 1971; Reimer, 1971; Snyder, 1970), incluso en organizaciones fuera del ámbito educativo; de hecho, se puede asociar incluso con la cultura de la organización si ésta no se manifiesta de forma explícita (Whitty et al., 1999).

En la Educación superior se han realizado distintos estudios relacionados con el impacto que el Curriculum Oculto tiene en la misma. Entre ellos, destacamos por su relevancia el 
realizado por Bergenhenegouwen (1987) que desarrolló dos proyectos de investigación relacionados con el impacto del Curriculum Oculto con los estudios relacionados con las Ciencias Sociales. En concreto, los estudios se centraron en los siguientes aspectos tales como la motivación y la actitud hacia el estudio.

Este autor sostiene que existe una tendencia entre los estudiantes para estudiar no sólo por conseguir un título (valor de cambio), sino también para integrar el estudio dentro sus vidas personales y encontrar un enlace con su propia experiencia cotidiana (valor práctico).

Por su carácter multidisciplinar cabría destacar el trabajo de Margolis (2002) que abarca cuestiones como mecanismos para ocultar y aflorar aspectos relacionados con el Curriculum, la educación en el Aula, la socialización de Capitalismo, la infiltración de la industria farmacéutica, el racismo, la desigualdad de género o la flexibilización del individuo en época de incertidumbre.

Además, existen varios trabajos relacionados con el curriculum oculto y la Universidad, fundamentalmente centrados en las áreas sociales y de la salud (Pujal et al., 2000; Rojas, 2012).

Por su relación con el propósito de este trabajo, queremos resaltar el trabajo elaborado por Moreno (2005) en el que analiza, desde una perspectiva teórica, los mecanismos socioeducativos en los que se produce la transmisión del Currículum Oculto a través de la práctica docente, en el contexto del cambio educativo experimentado por las sociedades occidentales. Para ello estudia el entramado sociocultural en el que se encuentra inmerso el docente, el cual explica en parte la forma en que se reproduce la transmisión del Currículum Oculto. En definitiva, el propósito de este artículo es fundamentar cómo los agentes socializadores implicados en la práctica docente universitaria contribuyen a la creación y transmisión de un Currículum Oculto que influye en el ejercicio docente y en la propia transmisión de conocimientos y creación de valores.

\section{EXPERIENCIA DESARROLLADA}

A la hora de poder explicar de manera razonable el influjo del Curriculum Oculto en la experiencia que vamos a describir resulta imprescindible contextualizar la situación en la que se desarrollará la experiencia, de modo que se entienda de qué modo ha podido influir en los procesos de toma de decisiones el Curriculum Oculto. Una vez realizada la contextualización del caso, procederemos a definir el elemento en el que se detecta la influencia del Curriculum Oculto para, finalmente, extraer una serie de conclusiones.

\subsection{Contextualización.}

La experiencia se realiza utilizando dos asignaturas optativas que se imparten en titulaciones diferentes: Gestión de Costes Laborales (en adelante, GCL-FICO), que se imparte en el Grado de Finanzas y Contabilidad y Contenidos Económicos para la Negociación Colectiva (en adelante, CENC-RRLL) impartida en el Grado de Relaciones Laborales y Recursos Humanos.

Ambas asignaturas tienen los siguientes rasgos:

a) Son asignaturas que se imparten en el segundo cuatrimestre del cuarto curso, último de la respectiva titulación.

b) Tienen 6 créditos ECTS.

c) Son asignaturas atípicas puesto que su organización corresponde a dos áreas de conocimiento diferentes (Economía Financiera y Contabilidad y Derecho del Trabajo y de la Seguridad Social), representadas por dos Departamentos: 
Contabilidad y Economía Financiera y Derecho del Trabajo y de la Seguridad Social, por lo que, en teoría, corresponde a cada área de conocimiento impartir 3 créditos ECTS.

d) Se imparten los mismos contenidos. Básicamente, se trata de analizar desde un punto de vista contable (para la gestión) las condiciones de trabajo, que pueden ser objeto de negociación colectiva, y que implican coste de personal. Desde un punto de vista jurídico se va a estudiar el régimen jurídico de cada una de estas condiciones (tiempo de trabajo y su distribución, salario y sistemas de retribución o clasificación profesional y movilidad).

e) Durante todos los cursos impartidos (2012-13 a 2020-21), el equipo de profesores ha sido el mismo en GCL-FICO y ha sido el mismo que ha impartido la asignatura de CENC-RRLL excepto en el curso 2014-15 donde hubo un cambio de docente procedente del Departamento de Contabilidad.

f) Comparten el mismo sistema de evaluación continua, del cual nos extenderemos a continuación.

Entre las diferencias entre ambas asignaturas señalamos las siguientes:

a) Los alumnos parten de un Currículum Formal diferente, lo que resulta evidente habida cuenta que las titulaciones tienen planes de estudios diferenciados y orientados a objetivos diferentes. Ello conlleva una profunda asimetría en el número de créditos relacionados con las materias relacionadas con las áreas de conocimiento implicadas en las respectivas asignaturas. En el cuadro $n^{\circ} 1$ vemos el número de créditos relacionados con las áreas de conocimiento y las distintas titulaciones:

Cuadro 1. Comparación del peso absoluto y relativo de las enseñanzas de derecho y contabilidad en los grados implicados (nota final 1)

\begin{tabular}{|c|c|c|}
\hline & RRLL & $\mathrm{FICO}$ \\
\hline CRÉDITOS EN DERECHO & 120 & 21 \\
\hline CRÉDITOS EN DERECHO DEL TRABAJO & $84(1)$ & 3(2) \\
\hline \% DERECHO DEL TRABAJO SOBRE TOTAL DERECHO & $70 \%$ & $14,29 \%$ \\
\hline \% DERECHO DEL TRABAJO SOBRE TOTAL TITULACIÓN & $34,15 \%$ & $1,35 \%$ \\
\hline CRÉDITOS EN CONTABILIDAD & 15 & 87 \\
\hline CRÉDITOS EN CONTABILIDAD PARA LA GESTIÓN & $3(3)$ & $21^{(4)}$ \\
\hline \% CONTABILIDAD PARA LA GESTIÓN S/ TOTAL CONTABILIDAD & $20 \%$ & $24,14 \%$ \\
\hline \% CONTABILIDAD PARA LA GESTIÓN S/ TOTAL TITULACIÓN & $1,22 \%$ & $9,46 \%$ \\
\hline
\end{tabular}

Fuente: elaboración propia a partir de los planes de estudio de las titulaciones.

Notas: ' Siempre que se curse la asignatura optativa Derechos Laborales de los Inmigrantes en la Unión Europea. De lo contrario, quedarían 78 Créditos Obligatorios.

2 Siempre que se curse la asignatura optativa Gestión de Costes Laborales, De lo contrario, el estudiante no recibiría formación alguna relacionada con el Derecho del Trabajo y de la Seguridad Social.

3 Siempre que se curse la asignatura optativa Contenidos Económicos de la Negociación Colectiva. De lo contrario, el estudiante no recibiría ninguna formación relacionada con la Contabilidad para la Gestión.

${ }^{4}$ Siempre que se cursen las asignaturas optativas Gestión de Costes Laborales y Control de Gestión. De lo contrario, quedarían en 12 Créditos obligatorios.

b) Diferencias en el Currículum Real: a pesar de que las asignaturas tienen los mismos contenidos, la desproporción que se manifiesta en el cuadro 1 hace que la profundidad con la que se estudian los contenidos (es decir, lo que realmente se le enseña al alumno) difiere de una asignatura a otra. Así, mientras que los contenidos relacionados con el área de conocimiento de Derecho del Trabajo y 
de la Seguridad Social en CENC-RRLL en una buena parte son un repaso general de lo que el estudiante de Relaciones Laborales ha ido recibiendo durante su formación académica, esas mismos contenidos resultan esenciales en GCL-FICO para comprender los fundamentos básicos del funcionamiento de las relaciones laborales en general y, en concreto, de los procesos que se generan durante una negociación colectiva. A sensu contrario, buena parte de los contenidos relacionados con la Contabilidad para la Gestión impartidos durante la asignatura GCL-FICO hacen referencia a elementos ya vistos en asignaturas precedentes y se ven de soslayo. Tanto en un supuesto como en otro los conceptos básicos de cada una de las materias que conforman las asignaturas se estudian de forma rápida a modo de recordatorio para, de este modo, poder introducir al alumno en los contenidos verdaderamente relevantes de la asignatura sin obviar los contenidos previamente aprendidos durante la carrera. El hecho que determinados contenidos sean vistos de manera rápida no significa en modo alguno que todos se vean de la misma forma sino que se ven como introducción a los contenidos verdaderamente relevantes para cada grupo en función de los contenidos previamente aprendidos.

\subsection{El sistema de evaluación y la detección del Currículum Oculto.}

El sistema de evaluación de la asignatura se basa en una evaluación continua del alumno basado en tres pilares:

a) Asistencia participativa a las clases. La participación activa de los estudiantes se realiza a través de los debates que se generan durante las exposiciones, respondiendo a las preguntas formuladas por el docente o cualquier otra forma de actitud proactiva (comentarios de noticias publicadas en prensa, búsqueda de información en páginas web de empresas sobre políticas de personal, sentencias relevantes, etc.), todas ellas forman parte de la evaluación.

b) Pruebas teóricas, prácticas o teórico-prácticas durante el curso a la finalización de un bloque temático relevante. Habida cuenta que la evaluación es continua, los contenidos evaluados no se liberan de posteriores evaluaciones, por lo que la construcción del conocimiento es acumulativo. Generalmente, se emplean las mismas pruebas para evaluar los conocimientos en ambas asignaturas.

c) Un trabajo en grupo. Próximo a la finalización del curso los alumnos, agrupados, deben aplicar los conocimientos adquiridos a un caso real. Para su materialización el grupo dispone de un convenio colectivo provincial de sector (hasta el momento, del sector hotelero) seleccionado y entregado por los docentes y de las cuentas anuales de una empresa, a elección del grupo, que esté o pueda incluirse en el ámbito de aplicación del convenio colectivo que se les ha facilitado. El objetivo del trabajo es la mejora de los resultados de la empresa a través de la elaboración de un convenio colectivo para su empresa, en el que se flexibilicen, dentro de los límites de la legislación vigente, las condiciones de trabajo previstas en el convenio colectivo de sector de ámbito provincial (tiempo de trabajo, remuneración o clasificación profesional). Posteriormente deben analizar el impacto económico de estas modificaciones mediante el análisis Coste-Volumen-Beneficio.

Este trabajo está sujeto a una serie de restricciones consistentes en el mantenimiento de la plantilla y la no utilización de los supuestos legales en los que por razones técnicas, económicas, organizativas o de producción, queda justificada una toma de decisión unilateral del empresario para realizar modificaciones sustanciales de condiciones de trabajo, traslados, expediente de regulación temporal de empleo o despido colectivo, 
por lo que las cuentas de las empresas no deben reunir esas condiciones económicas justificativas.

La flexibilidad es una herramienta que el legislador introduce en la normativa laboral para, entre otras razones, facilitar el ejercicio del derecho de libertad de empresa del empresario y, dentro de este su poder de organización y dirección.

Es en el alcance y contenido de estas medidas flexibilizadoras donde se observa como los estudiantes en el desarrollo de sus competencias a través de la simulación de una negociación colectiva adoptan medidas pro-empresario, pro-trabajador en función de que se traten de estudiantes de GCL-FICO o de CENC-RRLL, respectivamente.

En la realización de los trabajos los docentes, de manera conjunta y presencial, asesoran a los grupos de trabajo en cuestiones relacionadas con los objetivos y la metodología del trabajo, pero no en la realización de propuestas y medidas a adoptar para conseguir dichos objetivos, que son fruto exclusivo del trabajo en grupo.

Durante los años que llevan impartiéndose estas asignaturas, se ha mantenido una trayectoria similar pese a los esfuerzos de los docentes por intentar objetivar la adopción de unas medidas y condiciones de trabajo desde la negociación en la que ambas partes deben ceder para la consecución de un objetivo, que no es otro, que establecer el mayor equilibrio posible entre los intereses de la empresa y de los trabajadores.

Durante la presentación de los trabajos, es obligatoria la participación de cada uno de los componentes del grupo y es durante la exposición y defensa de los mismos, donde se manifiesta el currículum oculto en forma de razonamiento, propuestas tendentes a dar solución al problema plantado o de la forma de elaborar razonamientos destinados a responder preguntas formuladas bien por los docentes, bien por sus propios compañeros.

\section{RESULTADOS.}

Serían muchos y diversos los ejemplos que podemos traer a este trabajo, no obstante, al reducirse el estudio de las condiciones de trabajo a tiempo de trabajo, clasificación profesional, remuneración y movilidad funcional, las medidas a adoptar por los estudiantes inciden en la mayor parte de las ocasiones en los mismos aspectos, aunque eso sí, de forma más o menos intensa, porcentualmente hablando, en el tiempo de trabajo y en los conceptos retributivos.

Para facilitar la lectura del lector vamos a partir de la regulación de la condición de trabajo prevista en el TRET, un breve comentario sobre la posibilidad de flexibilización, para posteriormente incluir el tratamiento que de la misma realizan ambos tipos de estudiantes. El sector de producción sobre el que se ha trabajado ha sido el de la hostelería, utilizando como base el convenio colectivo de ámbito nacional vigente para el curso corriente.

Comenzamos por alguno de los aspectos de la regulación de la jornada, la jornada irregular y la organización del tiempo de trabajo.

Con carácter previo al análisis del supuesto concreto, es necesario acercarnos a la normativa general, como paso previo para entender las posibles interpretaciones que sobre la materia han realizado cada uno de los grupos y titulaciones.

Según el Texto Refundido del Estatuto de los Trabajadores la duración máxima de la jornada ordinaria de trabajo es de 40 horas semanales de trabajo efectivo de promedio en cómputo anual (1.826 h). Su duración viene concretada fundamentalmente por sus límites: diariamente la duración de la jornada ordinaria y regular de trabajo no puede exceder de 9 horas diarias existiendo la obligación de respetar, como mínimo 12 horas entre la finalización de una jornada y el comienzo de la siguiente. 
Dentro de la regulación anterior, los elementos más relevantes son la cuantificación anual de la jornada y el límite de descanso entre jornadas. Esta previsión permite, entre otras opciones organizativas por parte del empresario, la existencia de la jornada ordinaria irregular de trabajo que, como se indica a continuación, permite al empresario incrementar la duración de la jornada ordinaria de trabajo diaria hasta un $10 \%$ del total de la jornada anual por cada trabajador (182 h), si no se regula un porcentaje mayor o menor en la negociación colectiva, con el único límite de respetar el descanso mínimo entre jornadas.

\begin{tabular}{|c|c|}
\hline \multicolumn{2}{|c|}{ JORNADA IRREGULAR } \\
\hline \multicolumn{2}{|l|}{ Normativa } \\
\hline \multicolumn{2}{|c|}{$\begin{array}{l}\text { Respecto a la jornada irregular el Artículo 34.2. TRET establece que "Mediante } \\
\text { convenio colectivo o, en su defecto, por acuerdo entre la empresa y los } \\
\text { representantes de los trabajadores, se podrá establecer la distribución irregular de la } \\
\text { jornada a lo largo del año. En defecto de pacto, la empresa podrá distribuir de } \\
\text { manera irregular a lo largo del año el diez por ciento de la jornada de trabajo". } \\
\text { "... En defecto de pacto, las diferencias derivadas de la distribución irregular de la } \\
\text { jornada deberán quedar compensadas en el plazo de doce meses desde que se } \\
\text { produzcan". } \\
\text { Es necesario conocer esta distribución con un preaviso mínimo de cinco días, por lo } \\
\text { que podrá verse ampliado, como garantía organizativa para el trabajador, por el } \\
\text { convenio colectivo. }\end{array}$} \\
\hline Ejemplos de tratamiento en GCL-FICO & Ejemplos de tratamiento en CENC-RRLL \\
\hline $\begin{array}{l}\text { Ejemplo } 1 \text {. Distribución irregular, en función } \\
\text { de las necesidades económicas, técnicas, } \\
\text { de producción y organizativas de la } \\
\text { empresa, desde un mínimo del } 10 \% \text { y un } \\
\text { máximo del } 50 \% \text { de la jornada de trabajo a } \\
\text { lo largo del año. Es decir, se reordenará la } \\
\text { jornada de trabajo de los trabajadores o } \\
\text { puestos de trabajo que considere el jefe de } \\
\text { departamento, en función de las } \\
\text { necesidades del mismo. Consecuencias en } \\
\text { las variables del análisis C-V-B: sólo } \\
\text { afectará a las unidades de venta, } \\
\text { producción o rendimiento, que se verán } \\
\text { incrementadas. Esto provocaría un } \\
\text { aumento de los beneficios, puesto que los } \\
\text { costes fijos o variables no sufrirán variación } \\
\text { alguna. } \\
\text { Ejemplo 2. La jornada de trabajo se } \\
\text { establece en } 40 \text { horas semanales en } \\
\text { cómputo anual. La empresa podrá } \\
\text { distribuir irregularmente la jornada de } \\
\text { trabajo en un máximo del } 25 \% \text { (a diferencia } \\
\text { del } 10 \% \text { que marca la ley), donde obtener } \\
\text { una mayor flexibilidad en la jornada. }\end{array}$ & $\begin{array}{l}\text { Se establece una bolsa de } 180 \text { horas de } \\
\text { trabajo irregular, que se podrá aplicar } \\
\text { únicamente durante los meses de } \\
\text { marzo, abril, mayo, septiembre, octubre } \\
\text { y noviembre, en jornada de } 10 \text { horas } \\
\text { diarias como máximo y } 48 \text { horas } \\
\text { semanales en prolongación de jornada. } \\
\text { El resto del año, la jornada tendrá una } \\
\text { duración máxima de } 40 \text { horas } \\
\text { semanales, con una duración máxima } \\
\text { de } 9 \text { horas diarias. Dichas horas } \\
\text { irregulares se controlarán por escrito, a } \\
\text { fin de que empresas y trabajadores } \\
\text { puedan computarlas y habrán de ser } \\
\text { comunicadas previamente y por escrito } \\
\text { al trabajador. Dichas horas se } \\
\text { compensarán de mutuo acuerdo entre } \\
\text { las partes, bien por reducción de } \\
\text { jornada o en descansos. }\end{array}$ \\
\hline
\end{tabular}


Como comentarios, a efectos de organización, esta distribución es positiva para el empresario en la medida en la que puede incrementar la duración de la jornada diaria de sus trabajadores en función de sus necesidades productivas, solo preavisando con 5 días al trabajador sobre la modificación de su jornada habitual. Resulta evidente que la posibilidad que la norma brinda al empresario para flexibilizar el tiempo de trabajo, incrementando o disminuyendo la jornada de sus trabajadores durante un año natural, conlleva un perjuicio para el trabajador con relación a la propia organización de su vida.

Podemos observar que, el sesgo que se observa entre los estudiantes de $4^{\circ}$ curso de la asignatura de GCL-FICO incide en incrementar, en el convenio colectivo negociado entre ellos, el porcentaje a aplicar en la jornada irregular, lo que implica un incremento de horas considerable a disposición del empresario con la única obligación del preaviso y del descanso entre jornadas. Esta posibilidad en el sector de la hostelería, en una región como Andalucía facilita, sobre todo en temporada alta, la realización de un importante número de horas diarias sin que adquieran la consideración de extraordinarias. Esta realidad puede llegar a provocar que, en aras a respetar el descanso entre jornadas, los turnos hayan de alternarse lo que, además de alterar cualquier organización del tiempo libre del trabajador puede llegar a incidir muy directamente en su seguridad y salud en el trabajo.

Por el contrario, el tratamiento que sobre la jornada irregular realizan los estudiantes de CENC-RRLL va en un sentido totalmente diferente. Las opciones legales a su regulación por convenio colectivo hacen que prácticamente todos los grupos regulen la jornada irregular, pero de forma protrabajador y no proempresario. Los que son más proclives a flexibilizar esta condición de trabajo mantienen la regulación legal prevista del $10 \%$ de disponibilidad pero, o bien incrementan de forma considerable el tiempo de preaviso ampliándolo hasta desde 15 días hasta un mes, o bien prevén un plus salarial para los trabajadores afectados por la jornada irregular, opciones no contempladas por la ley, pero tampoco impedidas. La razón se encuentra en la necesidad de dar un margen suficiente al trabajador para que esta ampliación de jornada le perjudique lo menos posible, tanto en la organización de su tiempo libre como en su salud o bien que le suponga un incremento salarial que, pueda compensar la afectación de su tiempo libre. Una opción distinta es la adoptada por aquellos grupos que reducen el porcentaje de horas a disposición del empresario, normalmente se remiten a la legislación anterior previendo un $5 \%$ de disposición por parte del empresario del total de la jornada anual o, los grupos más protrabajador, mantienen esta opción, pero la causalizan, es decir, solo la contemplan si el empresario alega razones técnicas, económicas, organizativas o productivas, negándola en cualquier otro supuesto.

Para estos estudiantes de CENC-RRLL, la jornada irregular se presenta como una medida altamente perjudicial para los intereses de los trabajadores de ahí su limitación o el incremento de garantías para su implantación, mientras que para los estudiantes de GCL-FICO la regulación de la jornada irregular la contemplan como una de las herramientas de flexibilidad más idóneas para alcanzar el mayor nivel de rentabilidad en la utilización de los recursos humanos de la empresa. 


\section{Normativa}

\section{ORGANIZACIÓN DEL TIEMPO DE TRABAJO}

El artículo 34.8 TRET establece que "Las personas trabajadoras tienen derecho a solicitar las adaptaciones de la duración y distribución de la jornada de trabajo, en la ordenación del tiempo de trabajo y en la forma de prestación, incluida la prestación de su trabajo a distancia, para hacer efectivo su derecho a la conciliación de la vida familiar y laboral. Dichas adaptaciones deberán ser razonables y proporcionadas en relación con las necesidades de la persona trabajadora y con las necesidades organizativas o productivas de la empresa.

En la negociación colectiva se pactarán los términos de su ejercicio, que se acomodarán a criterios y sistemas que garanticen la ausencia de discriminación, tanto directa como indirecta, entre personas trabajadoras de uno y otro sexo. En su ausencia, la empresa, ante la solicitud de adaptación de jornada, abrirá un proceso de negociación con la persona trabajadora durante un periodo máximo de treinta días. Finalizado el mismo, la empresa, por escrito, comunicará la aceptación de la petición, planteará una propuesta alternativa que posibilite las necesidades de conciliación de la persona trabajadora o bien manifestará la negativa a su ejercicio. En este último caso, se indicarán las razones objetivas en las que se sustenta la decisión".

\section{Ejemplos de tratamiento en GCL-FICO}

Ejemplo 1. El trabajador podrá adaptar su horario por conciliación familiar cuando el empresario cuente con personal suficiente en ese servicio y pueda cubrir las diferencias horarias. De esa forma la actividad productiva no se verá afectada porque un trabajador entre más tarde o salga antes de su puesto de trabajo.

Ejemplo 2. El empresario puede alterar la distribución prevista en este convenio referida a los turnos, horario de entrada y salida (con un margen de una hora), cuando lo considere necesario sin necesidad de justificación. Cualquier alteración que suponga más de una hora, sí deberá justificarla.

\section{Ejemplos de tratamiento en CENC-RRLL}

Ejemplo 1. Los trabajadores encuadrados en los Niveles salariales I, II, III, IV, V, VI podrán flexibilizar su horario de forma que, respetando la jornada máxima anual, puedan modificar sus tiempos de trabajo y descanso atendiendo a los ciclos y necesidades específicos del puesto o coordinándolos con otros trabajadores de su misma responsabilidad en el área, siempre que quede garantizada una correcta atención a los objetivos del puesto.

Ejemplo 2. Normalmente solo se prestará el trabajo corriente. No obstante, temporalmente y por necesidad urgente de prevenir males 0 de remediar accidentes o daños sufridos, deberá el trabajador prestar mayor trabajo $\mathrm{u}$ otro distinto del acordado, con obligación por parte del empresario de indemnizar de acuerdo con la normativa aplicable al respecto.

En el caso de esta cuestión, la organización del tiempo de trabajo corresponde al empresario en función de su poder de dirección, pero el legislador también reconoce a los trabajadores un derecho a solicitar su adecuación en función de sus necesidades de conciliación familiar.

Como se observa en este precepto, el ejercicio de este derecho por parte del trabajador está condicionado al procedimiento previsto en la negociación colectiva y, en su defecto al acuerdo que pueda alcanzarse entre el empresario y el trabajador. Si le deniega su ejercicio o no se adecúa directamente a las necesidades del trabajador 
a este solo le queda la posibilidad de acudir a los tribunales de justicia para que se pronuncien sobre las razones aducidas por el empresario y sobre el respeto de su derecho. Los estudiantes han utilizado el amplio margen que el legislador atribuye a la negociación colectiva para en sus respectivos trabajados ampliar o limitar el propio poder organizativo del empresario, en el caso de los estudiantes de GCL-FICO, la condición para reconocerlo es que el puesto de trabajo pueda estar siempre cubierto con otros trabajadores. Para los Estudiantes de CENC-RRLL la decisión y la organización recae en manos del trabajador siempre que se cumpla el mismo objetivo, la cobertura del puesto de trabajo.

\begin{tabular}{|c|c|}
\hline \multicolumn{2}{|c|}{ HORAS EXTRAORDINARIAS } \\
\hline \multirow{2}{*}{\multicolumn{2}{|c|}{$\begin{array}{l}\text { Normativa } \\
\text { Artículo 35.1 TRET 1. Tendrán la consideración de horas extraordinarias aquellas horas } \\
\text { de trabajo que se realicen sobre la duración máxima de la jornada ordinaria de } \\
\text { trabajo, fijada de acuerdo con el artículo anterior. Mediante convenio colectivo o, } \\
\text { en su defecto, contrato individual, se optará entre abonar las horas extraordinarias en } \\
\text { la cuantía que se fije, que en ningún caso podrá ser inferior al valor de la hora } \\
\text { ordinaria, o compensarlas por tiempos equivalentes de descanso retribuido. En } \\
\text { ausencia de pacto al respecto, se entenderá que las horas extraordinarias realizadas } \\
\text { deberán ser compensadas mediante descanso dentro de los cuatro meses siguientes } \\
\text { a su realización. }\end{array}$}} \\
\hline & \\
\hline Ejemplos de tratamiento en GCL-FICO & Ejemplos de tratamiento en CENC-RRLL \\
\hline $\begin{array}{l}\text { Ejemplo 1. Debido a la situación actual, las } \\
\text { horas extraordinarias serán compensadas } \\
\text { por vacaciones dentro de los } 6 \text { meses } \\
\text { siguientes a la realización de dichas horas } \\
\text { extraordinarias. Cuando el estado de } \\
\text { alarma finalice, el trabajador podrá } \\
\text { compensar sus horas extras por su } \\
\text { retribución correspondiente. }\end{array}$ & $\begin{array}{l}\text { Ejemplo 1. Se abonarán de } \\
\text { conformidad con la legislación vigente, } \\
\text { con un incremento del } 80 \% \text { sobre la hora } \\
\text { ordinaria, pudiendo también ser } \\
\text { compensadas con descansos, en } \\
\text { proporción de } 1 \text { hora igual a } 1 \text { hora y } 45 \\
\text { minutos. }\end{array}$ \\
\hline $\begin{array}{l}\text { Ejemplo 2. Las partes firmantes coinciden } \\
\text { en declarar que es interés prioritario de } \\
\text { ambas el considerar que el número de } \\
\text { horas extras realizadas sea el menor posible } \\
\text { y que las mismas tengan carácter de } \\
\text { estructurales o de fuerza mayor. Son horas } \\
\text { extraordinarias las que excedan de } 1.826 \\
\text { horas de trabajo al año. El valor de la hora } \\
\text { ordinaria será de un } 5 \% \text { más en vez del } 8 \% \\
\text { que se venía pagando la hora ordinaria. A } \\
\text { los efectos de lo dispuesto en el párrafo } \\
\text { anterior, no se computarán las horas } \\
\text { extraordinarias que hayan sido } \\
\text { compensadas mediante descanso dentro } \\
\text { del año natural en el que se efectúen. }\end{array}$ & \\
\hline $\begin{array}{l}\text { Ejemplo } 3 . \text { Las horas extraordinarias } \\
\text { estaban pactadas por convenio colectivo } \\
\text { en ser compensadas mediante descanso } \\
\text { en los cuatro meses siguientes de su } \\
\text { realización. Este tiempo se extenderá para } \\
\text { poder compensarlas dentro de los } 12 \\
\text { meses siguientes. }\end{array}$ & \\
\hline
\end{tabular}


En el caso de las horas extraordinarias se ha de destacar el carácter voluntario de las mismas, salvo que se prevea su obligatoriedad en convenio colectivo o sea necesaria su realización para prevenir o reparar siniestros y otros daños extraordinarios y urgentes. Así como que como se recoge en la norma, el valor de la hora extraordinaria ha de ser, como mínimo, igual al previsto para la hora ordinaria de trabajo.

Los estudiantes, según la titulación, convierten en una obligación la realización de estas horas extraordinarias e incrementan, en mayor o menor medida, su valor económico e incluso el tiempo para su compensación por descanso. Resulta evidente que a menor valor económico mayor perjuicio sufre el trabajador, no solo por la reducción de la cuantía a percibir, sino que la equiparación del valor de la hora extraordinaria con la hora ordinaria, facilita su realización sin ningún requisito adicional, más allá que la propia necesidad del empresario. La Ley impone el límite de 80 horas extraordinarias al año como máximo, pero no forman parte de este cómputo todas las que se compensen con descansos dentro de los cuatro meses siguientes a su realización, por lo que la duración de la jornada ordinaria más la extraordinaria puede llegar a incrementar la productividad sin un coste adicional importante para el empresario, pero sí puede llevar un coste personal importante para el trabajador si no se regula de forma pormenorizada el procedimiento $y$, en su caso acuerdo con el empresario, la compensación con descansos incluido. En caso contrario el carácter extraordinario de estas horas podría pasar a formar parte de una jornada "ordinaria" de trabajo. Igual ocurre cuando se dilata en el tiempo su compensación por descanso más allá de los 4 meses previstos por la norma, aunque en este supuesto, todo el exceso en el tiempo sí computará a efecto de las 80 horas como máximo para una jornada completa de trabajo.

\begin{tabular}{|c|c|}
\hline \multicolumn{2}{|c|}{ GRATIFICACIONES EXTRAORDINARIAS } \\
\hline \multicolumn{2}{|l|}{ Normativa } \\
\hline \multicolumn{2}{|c|}{$\begin{array}{l}\text { Artículo } 31 \text { TRET "El trabajador tiene derecho a dos gratificaciones extraordinarias al } \\
\text { año, una de ellas con ocasión de las fiestas de Navidad y la otra en el mes que se fije } \\
\text { por convenio colectivo o por acuerdo entre el empresario y los representantes legales } \\
\text { de los trabajadores. Igualmente se fijará por convenio colectivo la cuantía de tales } \\
\text { gratificaciones. } \\
\text { No obstante, podrá acordarse en convenio colectivo que las gratificaciones } \\
\text { extraordinarias se prorrateen en las doce mensualidades". }\end{array}$} \\
\hline Ejemplos de tratamiento en GCL-FICO & Ejemplos de tratamiento en CENC-RRLL \\
\hline 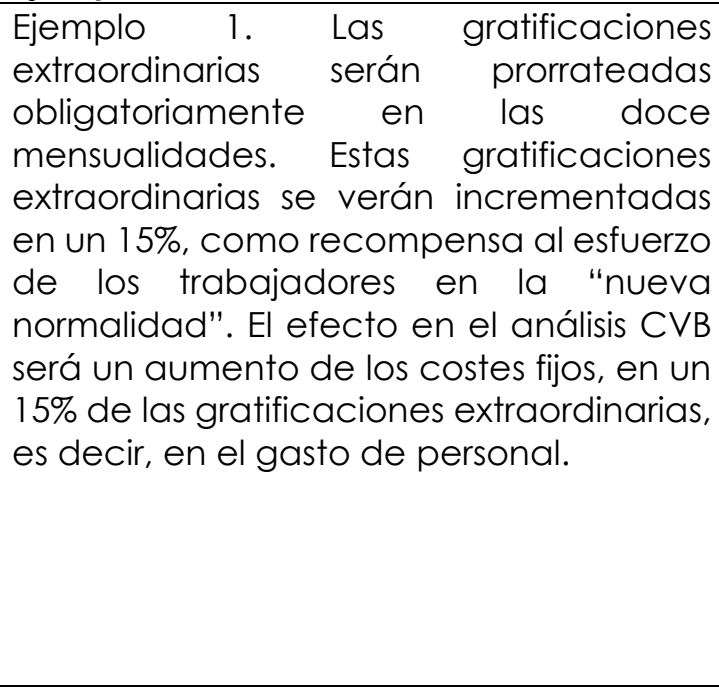 & $\begin{array}{l}\text { Ejemplo 1. Se establecen dos pagas } \\
\text { extras, que serán de treinta días cada } \\
\text { una y se devengarán de forma } \\
\text { prorrateada, por el importe del salario } \\
\text { base de este Convenio incrementado } \\
\text { con la antigüedad, generándose el } \\
\text { derecho a su percibo desde el día } \\
\text { siguiente de su devengo. } \\
\text { Se establece, además, una gratificación } \\
\text { en concepto de Paga de Primavera } \\
\text { equivalente a } 15 \text { días, igualmente del } \\
\text { salario base más antigüedad; su } \\
\text { cómputo será por años naturales y las } \\
\text { empresas la abonarán a sus trabajadores } \\
\text { antes del } 15 \text { de abril del año siguiente a } \\
\text { su devengo. }\end{array}$ \\
\hline
\end{tabular}

En el caso de las gratificaciones extraordinarias, las diferencias más importantes entre ambos grupos radican en la incorporación de una tercera gratificación denominada 
"de beneficios". En ambas titulaciones prevén que su importe está formado por el salario base y el complemento de antigüedad, aunque en el grupo de GCL-FICO el reconocimiento de esta gratificación y su cuantía se hace, sin embargo, para compensar de alguna forma la bajada generalizada todos los conceptos salariales del trabajador.

\begin{tabular}{|c|c|}
\hline \multicolumn{2}{|c|}{ SALARIO } \\
\hline \multicolumn{2}{|l|}{ Normativa } \\
\hline \multicolumn{2}{|c|}{$\begin{array}{l}\text { Artículo } 26.3 \text { TRET, Mediante la negociación colectiva o, en su defecto, el contrato } \\
\text { individual, se determinará la estructura del salario, que deberá comprender el salario } \\
\text { base, como retribución fijada por unidad de tiempo o de obra y, en su caso, } \\
\text { complementos salariales fijados en función de circunstancias relativas a las } \\
\text { condiciones personales del trabajador, al trabajo realizado o a la situación y } \\
\text { resultados de la empresa, que se calcularán conforme a los criterios que a tal efecto } \\
\text { se pacten. Igualmente se pactará el carácter consolidable o no de dichos } \\
\text { complementos salariales, no teniendo el carácter de consolidables, salvo acuerdo en } \\
\text { contrario, los que estén vinculados al puesto de trabajo o a la situación y resultados } \\
\text { de la empresa. }\end{array}$} \\
\hline Ejemplos de tratamiento en GCL-FICO & Ejemplos de tratamiento en CENC-RRLL \\
\hline $\begin{array}{l}\text { Ejemplo 1. Pasamos a analizar las } \\
\text { medidas propuestas de los } \\
\text { representantes de los trabajadores con } \\
\text { respecto a las retribuciones: } \\
\text { "Revisión salarial, solicitamos que para los } \\
\text { próximos } 4 \text { años se establezca una } \\
\text { revisión salarial de un punto por encima } \\
\text { del IPC anual, tomando como referencia } \\
\text { el IPC del mes de diciembre." } \\
\text { No procede aceptar esta medida. } \\
\text { "Plus fin de semana, solicitamos que, al } \\
\text { igual que existe un plus de turnicidad, se } \\
\text { establezca un plus de un } 15 \% \text { del valor de } \\
\text { la hora ordinaria cuando la jornada se } \\
\text { realice en fin de semana." } \\
\text { No procede aceptar esta medida. } \\
\text { - Se propone por parte de la empresa, las } \\
\text { siguientes propuestas a incluir: } \\
\text { - Se solicita establecer un periodo } \\
\text { formativo para cada grupo profesional } \\
\text { de } 6 \text { meses de duración, conllevando un }\end{array}$ & $\begin{array}{l}\text { Ejemplo } 1 . \\
\text { a. Plus Convenio. } \\
\text { Los trabajadores afectados por el } \\
\text { presente convenio percibirán el } \\
\text { denominado Plus Convenio, consistente } \\
\text { en } 10 \text { días de salario base mensual más } \\
\text { antigüedad. Dicho Plus se abonará el } 15 \\
\text { de octubre de cada año. } \\
\text { b. Plus de asistencia. } \\
\text { Los trabajadores afectados por el } \\
\text { presente convenio percibirán el } \\
\text { denominado Plus de Asistencia, el cual } \\
\text { será percibido en su integridad al } 100 \% \\
\text { por aquellos trabajadores, que por falta } \\
\text { injustificada y/o cuyo número de bajas } \\
\text { por IT sea inferior o igual a } 8 \text { días al año. } \\
\text { Se percibirá este Plus en un } 50 \% \text { cuando } \\
\text { concurran } 10 \text { faltas al año; y en la cuantía } \\
\text { del } 40 \% \text { cuando concurran } 11 \text { faltas al } \\
\text { año, no percibiendo en el supuesto de } 12 \\
\text { o más días de faltas al año. }\end{array}$ \\
\hline $\begin{array}{l}80 \% \text { de la retribución correspondiente a } \\
\text { cada grupo profesional. } \\
\text {-Se solicita una modificación en el salario } \\
\text { con una reducción del } 10 \% \text { en los grupos } \\
\text { profesionales } 2 \text { y } 3 \text {. } \\
\text { Se aceptan estas propuestas con el } \\
\text { compromiso de revisión en un año. }\end{array}$ & $\begin{array}{l}\text { cturnidad. } \\
\text { las en el período } \\
\text { as diez de la noche y } \\
\text { iana, tendrán una } \\
\text { a incrementada en } \\
\text { lario hora ordinario, }\end{array}$ \\
\hline $\begin{array}{l}\text { Ejemplo 2. Trabajador nocturno. La } \\
\text { retribución específica del trabajador } \\
\text { nocturno se renegocia a una reducción } \\
\text { del } 5 \% \text { de la remuneración por hora o se } \\
\text { compensarán mediante descanso a } \\
\text { elección del trabajador afectado. Esto }\end{array}$ & $\begin{array}{l}\text { parcial nocturna. } \\
\text { Los trabajadores cuya jornada sea } \\
\text { específicamente nocturna por su propia } \\
\text { naturaleza y dé comienzo con } \\
\text { posterioridad a las veintidós horas, } \\
\text { percibirán un plus del veinte por ciento }\end{array}$ \\
\hline
\end{tabular}


traerá como consecuencia la reducción del coste de personal.

Ejemplo 3.

a. Salario base. La cuantía del salario base de todos los trabajadores de la empresa sufrirán una reducción del $12 \%$ durante el año de vigencia de este convenio colectivo. El efecto en el análisis CVB una reducción del gasto de personal, y por lo tanto, de los costes fijos, aumentando las ganancias totales.

b. Complementos salariales. Se eliminarán o reducirán todos los complementos salariales percibidos por los trabajadores, durante el periodo de vigencia de este convenio colectivo, es decir, un año. Los complementos salariales de carácter consolidables, como la antigüedad, no computarán a efectos respecto a este año, recuperando su imputación normal a partir del año siguiente. El efecto en el análisis CVB será la disminución de los costes fijos $y$, como consecuencia, aumentarán las ganancias totales.

c. Anticipos a cuenta del trabajador. No se podrán realizar anticipos a cuenta del trabajo ya realizado. No afectaría a la variable coste volumen beneficio, pero puede mejorar la liquidez de la empresa. sobre el sueldo base establecido en este Convenio.

Ejemplo 3.

Artículo 22. Salarios. 1.Salario base. Los salarios para cada categoría profesional serán los que figuran en los Anexos I y II de este convenio. 2. Los incrementos económicos a todos los conceptos de este convenio serán los siguientes: a) Para el periodo comprendido entre 01/01/2020 a 31/12/2020 y sobre las tablas salariales vigentes en el año 2019 se establecerá un incremento del 2,65\% sobre todos los conceptos económicos recogidos en el convenio. (Anexo I). Artículo 23. Antigüedades. 1.- Los trabajadores que tuvieran hasta el 30/6/96 incrementos por antigüedad se respetarán en las cuantías que tuvieran establecidas hasta esa fecha y que tendrán el mismo incremento que el resto de los conceptos económicos del convenio. En caso de que esta cuantía no llegara al tope del $8 \%$ del SMI, se incrementará hasta dicho tope. 2.- Los trabajadores generaran el derecho por este concepto de antigüedad, hasta alcanzar como tope máximo el $8 \%$ sobre el Salario Mínimo Interprofesional (S.M.I) que por disposición del Gobierno rija en cada momento.

Atendiendo a la dicción de este precepto, y siempre desde el cumplimiento de la norma, solo tendrán la consideración de complementos salariales los que se prevean en el convenio colectivo y su concepto encaje perfectamente en alguno de los previstos en la tipología legal: 1) relativos a las condiciones personales del trabajador, 2) al trabajo realizado 3) o a la situación y resultados de la empresa.

Al no preverse ninguna cuantía, más allá del límite impuesto por la cuantía del salario mínimo interprofesional (SMI), la cantidad correspondiente al salario base vendrá determinado por el convenio colectivo en función de la clasificación profesional (grupos profesionales). Respecto a los complementos su cuantía podrá venir determinada por un porcentaje concreto del salario base o por una cantidad definida en el propio convenio.

La posibilidad de prever complementos en el convenio es muy utilizada por los estudiantes de ambas titulaciones aunque con evidentes diferencias cuantitativas y cualitativas ya que el número de complementos para los estudiantes del grado de Finanzas y Contabilidad se reducen de forma importante así como su revalorización, entrándose más en los personales y en la situación y resultados de la empresa, mientras que para los estudiantes del grado de Relaciones Laborales y Recursos Humanos incluyen todos los posibles incluso en ocasiones prevén pluses que en puridad no tienen la consideración de complementos, por lo que esas cantidades deberían ser consideradas como salario base. 
Se trata de una materia muy recurrente y profundamente regulada por los estudiantes en ambas titulaciones, por lo que solo se incluirán los aspectos más significativos referidos anteriormente.

$\mathrm{Si}$, como hemos visto anteriormente, existen muchos elementos en los que encontramos soluciones y puntos de vista divergentes, existen otros en los que hay coincidencia en la aplicación e interpretación de la norma. Aunque podríamos traer a este trabajo más ejemplos, hay uno que es muy significativo y que se extiende a todas las situaciones previstas por la ley en las que el ejercicio de un derecho no tiene la consideración de trabajo efectivo. Nos estamos refiriendo al descanso denominado coloquialmente como la pausa del "bocadillo" regulada en el artículo 34.4 TRET en el que se establece que: "Siempre que la duración de la jornada diaria continuada exceda de seis horas, deberá establecerse un periodo de descanso durante la misma de duración no inferior a quince minutos. Este periodo de descanso se considerará tiempo de trabajo efectivo cuando así esté establecido o se establezca por convenio colectivo o contrato de trabajo". Todos los grupos de estudiantes de ambas titulaciones han considerado este periodo de descanso como de trabajo efectivo, es decir, todos ellos entienden como derecho este tiempo y consideran que no debe ser compensado alargando la jornada. Las diferencias persisten, no obstante, cuando los estudiantes de GCL-FICO mantienen los 15 minutos legalmente previstos, mientras que los estudiantes de CENC-RRLL suelen ampliarlo a 30 minutos.

Igual podría decirse sobre la regulación de materias como los permisos retribuidos, las vacaciones o el descanso semanal, donde no se aprecian diferencias significativas de carácter interpretativo.

\section{CONCLUSIONES}

Una de las principales conclusiones de este estudio se basa en la percepción de un sesgo en los estudiantes de Finanzas y Contabilidad de un corte economicista, orientado a la consecución de objetivos aplicando soluciones donde primen, primordialmente, la eficacia, la eficiencia, la maximización del beneficio y el predominio del Análisis CosteVolumen-Beneficio como herramienta frente a la norma, a la que subordina. En cambio, en el Grado de Relaciones Laborales muestran una mayor preocupación por el cumplimiento de las normas, una visión más social donde las relaciones laborales y su defensa cobran un especial protagonismo, con independencia del impacto que sobre los resultados de la empresa puedan generarse.

En el caso de los alumnos de Gestión de Costes Laborales (GCL-FICO), se evidencia un claro sesgo a utilizar una mente economicista, basada en alcanzar la mejora de los resultados mediante la reducción de derechos de los trabajadores, empeorando sus condiciones de trabajo llegando, incluso, a situaciones hasta el momento ilegales (como la no contraprestación de horas extraordinarias, utilización de figuras de contratación temporal para luego aumentar el número de horas a trabajar, supresión de los descansos, de las vacaciones, entre otros).

Para los alumnos de Contenidos Económicos de la Negociación Colectiva (CENC-RRLL), también se evidencia un sesgo, más proclive a la defensa y mantenimiento de los derechos de los trabajadores sin atreverse, dentro de la legalidad, a utilizar instrumentos que, mejorando la eficiencia de la empresa, pueda afectar a esos derechos negativamente aun cuando utilizan medidas de flexibilidad, siempre buscan la forma de compensarlas incluso con beneficios que suponen, a la postre, un reducción de los resultados empresariales, cuando el objetivo del trabajo era la mejora de éstos.

Podría pensarse que la motivación de los estudiantes ante la elección de sus estudios ya viene condicionada por un interés previo y por su adecuación a una serie de actitudes y/o aptitudes que los definen, tratando de evitar aquellas titulaciones que no se 
adapten a ese encaje (Arquero et al., 2017). Esta apreciación se corresponde con el caso que nos ocupa ya que las asignaturas objeto de este análisis, en ambas titulaciones, suponen una disrupción en su programa formativo, aunque cada cual de un modo diferente, ya ambas tienen orientaciones diferentes, como se ha visto a lo largo de todo este trabajo, una materia que en el caso de GCL-FICO no se contempla en sus planes de estudios y en el caso de CENC-RRLL, aunque haya asignaturas de Contabilidad, no se estudia nada relacionado con estos aspectos. No obstante, y por esta razón, probablemente los estudiantes, conscientes de su carencia, prefieren optar por estas asignaturas.

A pesar de la existencia de una asignatura obligatoria en tercer curso del grado FICO denominada Responsabilidad Social y ética empresarial se observa cómo los objetivos de maximización del beneficio, de la eficiencia, de la rentabilidad de la empresa y del accionista que impregnan muchas de las asignaturas relacionadas con la Teoría Económica, la Contabilidad en sus distintos niveles y las Finanzas han condicionado notablemente a lo largo de su paso por la titulación la forma de razonar de los estudiantes. Por ejemplo, amplían el tiempo de trabajo (que, por sí solo, sería suficiente y justificado) pero, paralelamente, buscan una contraprestación que mejore la alteración de sus condiciones de trabajo anteriores.

Por su parte los alumnos del grado de Relaciones Laborales y Recursos Humanos, con una formación mucho más multidisciplinar, que abarca materias sociales, administrativas, organizativas de empresa y económicas se centra en aspectos más sociales en los que prima el interés (aparente) de trabajador frente al interés económico de la empresa. No obstante, estos estudiantes, en un conjunto significativo de casos, muestran aversión al uso de herramientas analíticas como, en nuestro caso, el instrumento de medición que se les ha explicado, el análisis Coste-Volumen-Beneficio, pese a que ello les reportaría una reducción de la calificación.

Si el objetivo de este trabajo fuera únicamente la identificación o confirmación de la existencia de un curriculum oculto, en ambas titulaciones los resultados obtenidos durante estos cursos, en las prácticas realizadas por nuestros alumnos confirmarían su existencia plenamente por lo que podríamos hablar de un éxito rotundo. Sin embargo, junto a la constatación de su existencia, hemos observado algo más y es la dificultad de cambio que se nos plantea en el aula. Tras el primer curso hemos intentado, utilizando los conocimientos adquiridos en otras asignaturas de cada uno de los grados y la experiencia adquirida con la asignatura, afianzar aquellos aspectos, que consideramos más positivos para cumplir con los itinerarios profesionales de los grados. El resultado aún no es positivo porque el sesgo fundamentalmente "economicista" o "social" adquirido durante la carrera sigue anclado en el antagonismo. Desde la diversidad, gestores financieros o gestores de recursos humanos, es necesario reforzar el curriculum real, de ambos grados, desde el desarrollo del curriculum formal y la integración del curriculum oculto como medio para poder dar una respuesta profesional adecuada a la empresa.

\section{LIMITACIONES Y POSIBLES EXTENSIONES DEL TRABAJO.}

Al tratarse de un estudio de caso, las conclusiones del trabajo estás circunscritas al entorno en el que se ha percibido el curriculum oculto. Por tanto, en otros centros y titulaciones similares es posible su detección y caso de que existiera, con distintos grados de intensidad respecto de los acreditados en este caso.

Una posible extensión del trabajo se puede centrar en someter a distintas pruebas a estudiantes de nuevo ingreso en titulaciones donde tienen un peso relativo importante las asignaturas de Derecho (Derecho, Relaciones Laborales, Criminología, Administración de Empresas y sus correspondientes dobles grados) junto con titulaciones 
donde ese peso relativo de las asignaturas de Derecho resulte poco significativa (p.e. Finanzas y Contabilidad) para conocer la influencia del curriculum oculto previo al comienzo de los estudios de modo que puedan ser sometidos a pruebas similares 0 idénticas en momentos previos al cierre de su expediente académico para comprobar el grado de influencia del currículum oculto en la resolución de los casos que se les planteen.

\section{REFERENCIAS}

Apple, M. W., \& King, N. R. (1977). What Do Schools Teach? Curriculum Inquiry, 6(4), $341-$ 358. https://doi.org/10.1080/03626784.1977.11075550

Arquero, J. L., Fernández-Polvillo, C., \& Valladares-García, D. (2017). Communication apprehension and students' educational choices: An exploratory analysis of Spanish secondary education students. Education and Training, 59(9), 946-956. https://doi.org/10.1108/ET-04-2017-0049

Bergenhenegouwen, G. (1987). Hidden curriculum in the university. Higher Education, 16(5), 535-543. https://doi.org/10.1007/BF00128420

Bonal, X. (2005). Sociología de la educación: una aproximación a las corrientes contemporáneas. Editorial Paidos.

Bowles, S., \& Gintis, H. (1976). Schooling in capitalist America Educational reform and the contradictions of economic life (Scientific Research Publishing (ed.)). Basic Books.

Carrillo Siles, B. (2009). Importancia del Curriculum Oculto en el proceso de EnseñanzaAprendizaje. Innovación y Experiencias Educativas, No 14, 1-10.

Casarini, M. (1999). Teoría y diseño curricular. Editorial Trillas.

Casarini, Martha. (2004). Teoría y Diseño Curricular. Editorial Trillas.

Celis, A., \& Moreno, A. (2017). La acriticidad e indiferencia también enseñan : curriculum oculto, outsourcing y degradación de las condiciones laborales. Revista Electrónica Sobre Cuerpos Académicos y Grupos de Investigación, 4 (8).

Dreeben, R. (1969). On What Is Learned In School. Educational Theory, 19(2), 214-220. https://doi.org/10.1111/j.1741-5446.1969.tb00386.x

Durkheim, E. (1961). Moral education a study in the theory and application of the sociology of education. Free Press of Glencoe.

Durkheim, E., Ortega, F., \& Manzano, P. (2002). La Educación moral. Ediciones Morata.

Etkin, J. R. (1993). La doble moral de las organizaciones: Los sistemas perversos y la corrupción institucionalizada. McGraw-Hill.

Flecha, R. (1999). Modern and Postmodern Racism in Europe: Dialogic Approach and Anti-Racist Pedagogies. Harvard Educational Review, 69(2), 150-172. https://doi.org/10.17763/HAER.69.2.3346055Q431G2U03

Giroux, H. (1992). Teoría y resistencia en Educacion (Vol. 112, Issue 483). Siglo xxi editores.

Illich, I. (1971). Deschooling Society. Harper \& Row.

Jackson, P. W. (1968). La vida en las aulas. Ediciones Morata.

Margolis, E. (2002). The Hidden Curriculum in Higher Education. In The Hidden Curriculum in Higher Education. https://doi.org/10.4324/9780203901854 
Moreno, A. (2005). La transmisión del currículum oculto en la práctica docente universitaria: contextos socioeducativos de la socialización. A modo de revisión teórica. El Guiniguada. Revista de Investigaciones y Experiencias En Ciencias de La Educación, 14(14), 177-190.

Moreno, E. (2000). La transmisión de modelos sexistas en la escuela. en Santos, M.A. El harén pedagógico: perspectiva de género en la organización escolar. Editorial Graó, Barcelona.

Posner, G. (2005). Analisis del curriculo. In McGraw Hill. McGraw-Hill México.

Pujal, M., Estrada, M., López, D., Montero, M., Pajares, A., Espinàs, L., \& Venegas, G. (2000). El currículum oculto en la Universidad: análisis psicosocial de algunas producciones académicas en Ciencias Sociales y de la Salud. In A. Ovejero, M. de la Villa, \& J. Vivas (Eds.), Aplicaciones de Psicología Social (p. 35). Biblioteca Nueva.

Reimer, E. (1971). An essay on alternatives in education. Interchange, 2(1), 1-35. https://doi.org/10.1007/BF02140862

Riseborough, G. F. (1988). Pupils, Recipe Knowledge, Curriculum and the Cultural Production of Class, Ethnicity and Patriarchy: A critique of one teacher's practices. British Journal of Sociology of Education, 9(1), 39-54. https://doi.org/10.1080/0142569880090103

Rojas O, A. (2012). "Curriculum oculto" en medicina: una reflexión docente. Revista Médica de Chile, 140(9), 1213-1217. https://doi.org/10.4067/S003498872012000900017

Santos Guerra, M. Á. (1996). Curriculum oculto y construcción del género en la escuela. Kikiriki. Cooperación Educativa, 42-43, 14-27.

Silverwood, V., Chew-Graham, C. A., Raybould, I., Thomas, B., \& Peters, S. (2017). BMC Medical Education. BMC Medical Education, 17. https://doi.org/10.1186/s12909017-0972-6

Snyder, B. R. (1970). The Hidden Curriculum. Routledge \& Kegan Paul.

Torres, J. (1998). El curriculum oculto. Ediciones Morata.

Whitty, G., Power, S., \& Halpin, D. (1999). La Escuela, el Estado y el Mercado. Ediciones Morata.

\section{Nota final}

1 Explicitados a través de los planes de estudios de las distintas titulaciones estudiadas en este trabajo como son:

Para el Grado en Finanzas y Contabilidad: https://www.us.es/estudiar/queestudiar/oferta-de-grados/grado-en-finanzas-y-contabilidad,

Para el grado en Relaciones laborales y Recursos Humanos: https://www.us.es/estudiar/que-estudiar/oferta-de-grados/grado-en-relacioneslaborales-y-recursos-humanos 